Faculty of Applied Mathematics

University of Twente

University for Technical and Social Sciences
P.O. Box 217

7500 AE Enschede

The Netherlands

Phone: +31-53-4893400

Fax: +31-53-4893114

Email:

memo@math.utwente.nl

Memorandum No. 1371

About convergence of numerical approximations

to homoclinic twist bifurcation points in

$Z_{2}$-symmetric systems in $\mathbb{R}^{4}$

S.A. VAN GILS AND V. TChistiakov

FEBRUARY 1997

ISSN 0169-2690 


\title{
About convergence of numerical approximations to homoclinic twist bifurcation points in $Z_{2}$-symmetric systems in $\mathbb{R}^{4}$.
}

\author{
S. A. van Gils \\ V.Tchistiakov \\ Faculty of Applied Mathematics \\ University of Twente
}

\begin{abstract}
An algorithm to detect homoclinic twist bifurcation points in $Z_{2}$ symmetric autonomous systems of ordinary differential equations in $\mathbb{R}^{4}$ along curves of symmetric homoclinic orbits to hyperbolic equilibria has been developed.We show convergence of numerical approximations to homoclinic twist bifurcation points in such systems. A test function is defined on the homoclinic solutions, which has a regular zero in the codimensiontwo bifurcation points. This codimension-two singularity can be continued appending the test function to a three parameter vector field. We demonstrate the use of the test function on several examples of two coupled Josephson junctions.
\end{abstract}

Key words. homoclinic twist bifurcation, $Z_{2}$-symmetry, continuation

AMS classification: 58F14 34C23 34C37 35B32 58F40

\section{Introduction}

In a two-parameter family of vector fields, there is generically a branch in the parameter plane along which orbits homoclinic to hyperbolic equilibria occur. Performing numerical experiments, codimension-two homoclinic orbits can be detected along branches of codimension-one homoclinics by locating zeros of a test function [4]. This paper presents an algorithm for numerical detection of homoclinic twist points in a two-parameter family of $Z_{2}$-symmetric vector fields 
in $\mathbb{R}^{4}$ with the help of a test function. The codimension-two singularity can be continued appending the test function to the problem in a three parameter vector field.

This codimension-two point can be characterized in the following way. Consider a unique, up to scalar multiplication, solution of the linearized flow around the homoclinic orbit whose direction limits in backward time on the most unstable direction of the saddle. In the forward time limit, this solution approaches precisely the same direction or exactly the opposite direction. It is shown that as parameters vary along the curve of homoclinic solutions a transition occurs between these two cases. At the transition point, solutions of the linearized flow around the homoclinic orbit whose direction limits in backward time on the most unstable direction of the saddle approach in the forward time limit the most stable direction. A point of the transition is called a homoclinic twist point.

A similar transition can occur in non-symmetric systems. Then it will be a codimension-three phenomenon. Thus test functions designed by Sandstede [10] for inclination bifurcations of vector fields without symmetry and then for general $n$-dimensional systems in [4] may not have meaning for symmetric systems with a homoclinic twist point. A test function for the homoclinic twist bifurcation in presence of symmetry has been derived for the first time.

The codimension-one homoclinic orbits have been computed using boundaryvalue methods, which have been recently analyzed by Beyn [3] and Schecter [11]. Such methods truncate the homoclinic problem to a finite interval with certain boundary conditions at the end points. In this paper, we prove a theorem about the rate of convergence of the computed parameter values for a codimension-two homoclinic orbit to the true parameter values as the length of the time interval on which the solution is computed increases.

The outline of the rest of the paper is as follows. In Section 1.1, we describe families of vector fields near a twist point as in [1]. In Section 2.1, we describe an algorithm for the computation of hyperbolic homoclinic orbits as in [11]. In section 2.2, we introduce a test function which is used to detect the homoclinic twist point and state the main result about convergence of the test function monitored along numerically computed homoclinic orbits on $\left[-T_{1}, T_{2}\right]$ as $T_{1}, T_{2} \rightarrow$ $\infty$. In Section 2.3, we prove the main result. Section 3 contains numerical analysis using the algorithm and results on a system of two coupled Josephson junctions. In Section 4, we show how the method for detecting homoclinic twist bifurcations 
can be extended to $\Gamma$-equivariant vector fields in $\mathbb{R}^{n}$, where $\Gamma$ is a finite subgroup of $O(n)$.

\subsection{Homoclinic twist point}

First, we formulate the twist bifurcation problem with $Z_{2}$ symmetry. We consider a family of ordinary differential equations

$$
\dot{x}=F(x, \alpha, \lambda),
$$

where $x(t) \in \mathbb{R}^{4}, \alpha, \lambda \in \mathbb{R}$ and $F: \mathbb{R}^{4} \times \mathbb{R}^{2} \rightarrow \mathbb{R}^{4}$ is a $C^{3}$ smooth function, $F(0, \alpha, \lambda)=0$ for all $\alpha, \lambda \in \mathbb{R}$.

$\left(H_{1}\right) F(\cdot, \alpha, \lambda)$ commutes with the action of $Z_{2}$ on $\mathbb{R}^{4}=S \oplus S^{\perp}$ given by: $\sigma y=y$, $y \in S$ and $\sigma y=-y, y \in R=S^{\perp}$, where $\sigma$ is the reflection in $Z_{2} ; \operatorname{dim} S=2$.

Symmetry implies that $D_{1} F\left(q_{\lambda}(t), 0, \lambda\right)$ commutes with $\sigma$ and can be written in appropriate symmetric coordinates in the following form:

$$
D_{1} F\left(q_{\lambda}(t), 0, \lambda\right)=\left(\begin{array}{cc}
\mathcal{A}_{\lambda}\left(q_{\lambda}(t)\right) & 0 \\
0 & \mathcal{B}_{\lambda}\left(q_{\lambda}(t)\right)
\end{array}\right),
$$

where $\mathcal{A}_{\lambda}\left(q_{\lambda}(t)\right), \mathcal{B}_{\lambda}\left(q_{\lambda}(t)\right)$ are $2 \times 2$ blocks. We write $\mathcal{A}_{\lambda}$ and $\mathcal{B}_{\lambda}$ for $\mathcal{A}_{\lambda}(p)$ and $\mathcal{B}_{\lambda}(p)$.

$\left(H_{2}\right)$ We assume that the eigenvectors of $D_{1} F(p, \alpha, \lambda)$ do not depend on $(\alpha, \lambda)$. For $\alpha=0, \lambda \in \Lambda=\left[\lambda_{1}, \lambda_{2}\right], \lambda_{1}<0<\lambda_{2}$, (1) has a smooth family of homoclinic orbits $q_{\lambda}(t)$ to a saddle $p=0$ and $q_{\lambda}(t) \in S=\operatorname{Fix}_{\mathbb{R}^{4}}\left(Z_{2}\right)$. Thus, $\mathcal{A}_{\lambda}$ has one negative eigenvalue $\sigma_{S}^{s}(\lambda)$ and one positive eigenvalue $\sigma_{S}^{u}(\lambda)$. We assume that $\forall \lambda \in \Lambda, \sigma_{S}^{s}(\lambda)<0$ and $\sigma_{S}^{u}(\lambda)>0$.

$\left(H_{3}\right) \mathcal{B}_{\lambda}$ has one positive and one negative eigenvalue $\sigma_{R}^{u}(\lambda)\left(\right.$ resp. $\left.\sigma_{R}^{s}(\lambda)\right)$ such that $\forall \lambda \in \Lambda, \sigma_{R}^{u}(\lambda)>0$ and $\sigma_{R}^{s}(\lambda)<0$, and none of the eigenvalues $\left\{\sigma_{S}^{s}(\lambda), \sigma_{S}^{u}(\lambda), \sigma_{R}^{s}(\lambda), \sigma_{R}^{u}(\lambda)\right\}$ have equal modulus for $\lambda \in \Lambda$.

$\left(H_{4}\right)$ The linear differential equation $\dot{y}=\mathcal{A}_{\lambda}\left(q_{\lambda}(t)\right) y$ has a unique, up to scalar multiplication, bounded solution $\dot{q}_{\lambda}$. Let $\phi(t)=\left(\begin{array}{c}\phi_{1}(t) \\ \phi_{2}(t)\end{array}\right)$ be the unique, 
up to scalar multiplication, bounded solution of the equation $\dot{\phi}=-\mathcal{A}_{0}^{T}\left(q_{0}(t)\right) \phi$ then

$$
\int_{-\infty}^{\infty} \psi(t) D_{2} F\left(q_{0}(t), 0,0\right) d t \neq 0
$$

where $\psi(t)=\left(\phi_{1}(t), \phi_{2}(t), 0,0\right)$.

Let $\chi_{S}^{u}, \chi_{S}^{s}$ be the unit length eigenvectors corresponding to the positive (negative) eigenvalue of $\mathcal{A}_{\lambda}$ and $\chi_{R}^{u}, \chi_{R}^{s}$ be the unit length eigenvectors corresponding to the positive (negative) eigenvalue of $\mathcal{B}_{\lambda}$.

Consider the linearized equation

$$
\dot{y}=D_{1} F\left(q_{\lambda}(t), 0, \lambda\right) y .
$$

For $t \rightarrow \pm \infty$, the behavior of its solutions is qualitatively the same as the behavior of the solutions of

$$
\dot{y}=D_{1} F(p, 0, \lambda) y .
$$

It follows that (2) has a unique solution, $y_{\lambda}$ such that $\lim _{t \rightarrow-\infty} \frac{y_{\lambda}(t)}{\left|y_{\lambda}(t)\right|}=\chi_{R}^{u}$ and $\left|y_{\lambda}(0)\right|=1$. Then, generically [1], $\lim _{t \rightarrow \infty} \frac{y_{\lambda}(t)}{\left|y_{\lambda}(t)\right|}= \pm \chi_{R}^{u}$.

Definition 1.1 If $\lim _{t \rightarrow \infty} \frac{y_{\lambda}(t)}{\left|y_{\lambda}(t)\right|}$ points in the opposite directions for $\lambda>0$ and $\lambda<0$ then we call the point $(\alpha, \lambda)=(0,0)$ a homoclinic twist point.

The point of transition $(\alpha, \lambda)=(0,0)$ can be characterized as follows. Let $W^{u}$ $\left(W^{u}\right)$ denote the unstable (resp. stable) manifold of $p$ for $\alpha=0, \lambda=0$. Let $T_{a} W^{u}\left(T_{b} W^{s}\right)$ denote the tangent space to $W^{u}$ at the point $a \in W^{u}$ (resp. the tangent space to $W^{s}$ at the point $\left.b \in W^{s}\right)$. The invariance of $S$ implies that at the point $(\alpha, \lambda)=(0,0)$ the vector $\chi_{R}^{u}$ is transported in forward time from $-\infty$ to $+\infty$ by the linearized equation into the direction of $\chi_{R}^{s}$. We conclude that the homoclinic orbit $q_{0}(t)$ is degenerate in the sense that

$\left(H_{5}\right) \operatorname{dim} T_{q_{0}(0)} W^{s} \cap T_{q_{0}(0)} W^{u}=2$.

As an example, we consider the following system of two coupled Josephson junctions in the case of a capacitive load written in symmetric coordinates [1]:

$$
\begin{aligned}
& \dot{s}_{1}=s_{2}, \\
& \dot{s}_{2}=\frac{1}{3+\beta}\left(I-s_{2}-\sin \left(s_{1}\right) \cos \left(r_{1}\right)\right), \\
& \dot{r}_{1}=r_{2}, \\
& \dot{r}_{2}=-\frac{1}{\beta}\left(r_{2}+\sin \left(r_{1}\right) \cos \left(s_{1}\right)\right) .
\end{aligned}
$$


The fixed point subspace of $Z_{2}$ is $S=\left\{\left(s_{1}, s_{2}, r_{1}, r_{2}\right) \in \mathbb{R}^{4}: r_{1}=r_{2}=0\right\}$ and $p=\left(\pi-\sin ^{-1}(I), 0,0,0\right)$ is a hyperbolic equilibrium.

D.G.Aronson, M.Golubitsky, M.Krupa [1] proved a theorem about existence of homoclinic orbits of (3) in $S$ and showed that after an appropriate change in the parameters and the state variables this system satisfies the hypotheses $H_{1}-H_{5}$.

\section{The Algorithm and the Theorem}

\subsection{Continuation of codimension-one homoclinic orbits}

Generic homoclinic orbits have been computed using boundary-value methods. Such methods truncate the homoclinic problem to a finite interval with certain boundary conditions at the end points.

We consider first (1). We wish to find all values of $(\alpha, \lambda)$ for which there is an orbit homoclinic to $p$. A homoclinic solution can be thought of as the solution of an infinite-interval boundary value problem. The boundary conditions are $x( \pm \infty)=p$. However, it is also a solution of a finite-interval boundary value problem. Let $W_{l o c}^{s}(p, \alpha, \lambda)\left(W_{l o c}^{u}(p, \alpha, \lambda)\right)$ denote the local stable (unstable) manifold of $p$, and let $J=\left[-T_{1}, T_{2}\right], T_{1}, T_{2}>0$. Then a solution of (1) in $S$ and homoclinic to $p$ satisfies the boundary conditions $x\left(-T_{1}\right) \in W_{l o c}^{u}(p, \alpha, \lambda) \cap S$, $x\left(T_{2}\right) \in W_{l o c}^{s}(p, \alpha, \lambda) \cap S$. Since a phase shift of the solution is again a solution, we must add a phase condition to resolve this redundancy. This is achieved by adding the condition in the form of a functional, defined on the solution $x$, $\Phi_{J}(x, \alpha, \lambda)=0$, where $\Phi_{J}: C^{0}\left(J, \mathbb{R}^{4}\right) \times \mathbb{R} \times \mathbb{R} \rightarrow \mathbb{R}$. Here we use the following phase condition: we fix the crossection $\Sigma=q_{0}(0)+\dot{q}_{0}(0)^{\perp}$, which is locally transverse to the flow for all $\lambda \in \Lambda$ and all small $\alpha$ and require that the solution intersects $\Sigma$ at time zero. We obtain the following finite-interval boundary value problem denoted by $P_{1}$ :

$$
P_{1}\left\{\begin{aligned}
\dot{x}(t) & =F(x(t), \alpha, \lambda), t \in J, \\
x\left(-T_{1}\right) & \in W_{l o c}^{u}(p, \alpha, \lambda) \cap S, \\
x\left(T_{2}\right) & \in W_{l o c}^{s}(p, \alpha, \lambda) \cap S \\
x(0) & \in \Sigma .
\end{aligned}\right.
$$

Given $\lambda$, and assuming $H_{1}-H_{4}$, a solution $\left(q_{\lambda}^{J}, \alpha_{\lambda}\right)$ of $P_{1}$ is locally unique in 
$C^{1}\left(J, \mathbb{R}^{n}\right) \times \mathbb{R}$. Our assumptions imply that $\alpha_{\lambda}=0$ for $\lambda \in \Lambda$. We can assume without loss of generality that $q_{\lambda}^{J}(t)=q_{\lambda}(t), t \in\left[-T_{1}, T_{2}\right]$ due to the fact that the phase condition is given in the form of a functional independent of $J$ and $(\alpha, \lambda)$.

In numerical computations, the local stable and unstable manifolds of $p$ must be approximated, say by $\tilde{W}^{s}(p, \alpha, \lambda)$ and $\tilde{W}^{u}(p, \alpha, \lambda)$. One then obtains a boundary value problem denoted by $P_{2}$ :

$$
P_{2}\left\{\begin{aligned}
\dot{x}(t) & =F(x(t), \alpha, \lambda), t \in J, \\
x\left(-T_{1}\right) & \in \tilde{W}^{u}(p, \alpha, \lambda) \cap S \\
x\left(T_{2}\right) & \in \tilde{W}^{s}(p, \alpha, \lambda) \cap S \\
x(0) & \in \Sigma
\end{aligned}\right.
$$

In order to compare solutions of the problems $P_{1}$ and $P_{2}$ we can apply the following theorem. Let $\epsilon>0$ and $\sigma_{S}^{s}, \sigma_{R}^{s}<0$ and $\sigma_{S}^{u}, \sigma_{R}^{u}>0$ be such that $\forall \lambda \in \Lambda$, $\sigma_{S}^{s}(\lambda)+\epsilon \leq \sigma_{S}^{s}, \sigma_{R}^{s}(\lambda)+\epsilon \leq \sigma_{R}^{s}, \sigma_{S}^{u}(\lambda)-\epsilon \geq \sigma_{S}^{u}$ and $\sigma_{R}^{u}(\lambda)-\epsilon \geq \sigma_{R}^{u}$.

Theorem 2.1 [Theorem 3.3 in [11]] Let $H_{1}-H_{4}$ hold and $\epsilon>0$. If $T_{1}, T_{2}$ are sufficiently large then $P_{2}$ has a unique solution $\left(\tilde{q}_{\lambda}^{J}, \tilde{\alpha}_{\lambda}^{J}\right)$. There is a constant $C$ which does not depend on $J$ and $\lambda$, such that the following estimates hold

$$
\begin{aligned}
\left|\tilde{\alpha}_{\lambda}\right| & \leq C\left(e^{-\left(-\sigma_{S}^{s}+2 \sigma_{S}^{u}\right) T_{1}}+e^{-\left(-2 \sigma_{S}^{s}+\sigma_{S}^{u}\right) T_{2}}\right) \\
\left|\tilde{q}_{\lambda}^{J}(t)-q_{\lambda}^{J}(t)\right| & \leq C\left(e^{-2 \sigma_{S}^{u} T_{1}}+e^{2 \sigma_{S}^{s} T_{2}}\right)
\end{aligned}
$$

Remark: The independence of $C$ of $\lambda$ is a consequence of $H_{2}$.

\subsection{Detection of the homoclinic twist point}

First, we compute a branch of symmetric homoclinic orbits. Then, we detect the homoclinic twist point using a test function which is monitored along this branch. In order to detect the homoclinic twist point, the truncated problem should be formulated in such a way that it can be continued through the degenerate point.

Consider the unique solution $u_{\lambda}(t)$ of the boundary value problem

$$
\left\{\begin{aligned}
\dot{u} & =\mathcal{B}_{\lambda}\left(q_{\lambda}(t)\right) u, \\
\lim _{t \rightarrow-\infty} \frac{u(t)}{|u(t)|} & =\chi_{R}^{u}, \\
|u(0)| & =1
\end{aligned}\right.
$$


and the unique solution $w_{\lambda}(t)$ of the boundary value problem

$$
\left\{\begin{aligned}
\dot{w} & =\mathcal{B}_{\lambda}\left(q_{\lambda}(t)\right) w \\
\lim _{t \rightarrow \infty} \frac{w(t)}{|w(t)|} & =\chi_{R}^{s} \\
|w(0)| & =1 .
\end{aligned}\right.
$$

It follows from $(5 \mathrm{a})$ and $(5 \mathrm{~b})$ that

$$
\begin{aligned}
& w_{\lambda}(t) \in T_{q_{\lambda}(t)} W^{s}(p, 0, \lambda), t \in \mathbb{R}_{+}, \\
& u_{\lambda}(t) \in T_{q_{\lambda}(t)} W^{u}(p, 0, \lambda), t \in \mathbb{R}_{-} .
\end{aligned}
$$

By $H_{5}$, at the twist point $u_{0}(0)= \pm w_{0}(0)$.

The defining equation for the twist bifurcation is given then by

$$
\left\langle u_{\lambda}(0), w_{\lambda}^{\perp}(0)\right\rangle=0 .
$$

We make the following assumption about the mapping

$$
\lambda \mapsto \tau(\lambda)=\left\langle u_{\lambda}(0), w_{\lambda}^{\perp}(0)\right\rangle
$$

$\left(H_{6}\right) \tau^{\prime}(0) \neq 0$.

Geometrically, $\left(H_{6}\right)$ means that the "angle" between the stable and unstable manifolds, which is zero at $\lambda=0$ has non-zero derivative there. Our numerical studies suggest that the system (3) satisfies $H_{6}$.

In order to obtain an approximation for the $\tau(\lambda)$, we replace (5a)-(5b) by the truncation, where $\left(q_{\lambda}(t), 0\right)$ is replaced by the solution $\left(\tilde{q}_{\lambda}^{J}(t), \tilde{\alpha}_{\lambda}^{J}\right)$ to the perturbed truncated problem with $\mathcal{B}_{\lambda}^{J}\left(\tilde{q}_{\lambda}^{J}(t)\right)=D_{1} F\left(\tilde{q}_{\lambda}^{J}(t), \tilde{\alpha}_{\lambda}, \lambda\right)$. Let $\tilde{y}_{\lambda}^{J}$ be the unique solution of the boundary value problem:

$$
\left\{\begin{aligned}
\dot{y} & =\mathcal{B}_{\lambda}^{J}\left(\tilde{q}_{\lambda}^{J}(t)\right) y, t \in\left[-T_{1}, 0\right] \\
\frac{y\left(-T_{1}\right)}{\left|y\left(-T_{1}\right)\right|} & =\chi_{R}^{u} \\
|y(0)| & =1
\end{aligned}\right.
$$

and $\tilde{z}_{\lambda}^{J}$ be the unique solution of the boundary value problem:

$$
\left\{\begin{aligned}
\dot{z} & =\mathcal{B}_{\lambda}^{J}\left(\tilde{q}_{\lambda}^{J}(t)\right) z, t \in\left[0, T_{2}\right] \\
\frac{z\left(T_{2}\right)}{\left|z\left(T_{2}\right)\right|} & =\chi_{R}^{s} \\
|z(0)| & =1
\end{aligned}\right.
$$


Evaluating the solutions at $t=0$, yields the following test function $\tau_{J}: \Lambda \rightarrow \mathbb{R}$ :

$$
\tau_{J}(\lambda)=\left\langle\tilde{y}_{\lambda}^{J}(0), \tilde{z}_{\lambda}^{J}(0)^{\perp}\right\rangle .
$$

The following theorem shows that the test function is well defined for both the original and the perturbed boundary value problems.

Theorem 2.2 Suppose that $H_{1}-H_{6}$ hold. For $\lambda \in \Lambda$, let $q_{\lambda}^{J}$ be solution of the problem $P_{1}$. Suppose we use linear approximations to $W_{\text {loc }}^{s}(p, \alpha, \lambda)$ and $W_{l o c}^{u}(p, \alpha, \lambda)$ denoted by $E^{s}(p, \alpha, \lambda)$ and $E^{u}(p, \alpha, \lambda)$. Then $\forall \epsilon>0$ there is a ball whose radius is independent of $J=\left[-T_{1}, T_{2}\right]$ and $\lambda$ about $\left(q_{\lambda}, 0\right)$ in $C^{0}\left(J, \mathbb{R}^{2}\right) \times \mathbb{R}$, and there are two positive constants $m$ and $M$ such that for $T_{1}, T_{2}$ sufficiently large:

(i) $\left(q_{\lambda}^{J}(t), 0\right)$ is the unique solution of $\left(P_{1}\right)$ in that ball;

(ii) the perturbed problem $\left(P_{2}\right)$ has a unique solution $\left(\tilde{q}_{\lambda}^{J}(t), \tilde{\alpha}_{\lambda}\right)$ in that ball;

(iii) $\exists \tilde{\lambda}$ such that $\left(\tilde{q}_{\tilde{\lambda}}(t), \tilde{\alpha}_{\tilde{\lambda}}\right)$ is the unique solution of $\left(P_{2}\right)$, that satisfies also $\tau_{J}(\tilde{\lambda})=0$. Moreover,

$$
|\tilde{\lambda}|+\left|\tilde{\alpha}_{\tilde{\lambda}}\right|=O\left(e^{\left(-\sigma_{R}^{u}+\sigma_{R}^{s}+\epsilon\right) T_{1}}+e^{\left(-\sigma_{R}^{u}+\sigma_{R}^{s}+\epsilon\right) T_{2}}+e^{-\left(2 \sigma_{s}^{u}-\epsilon\right) T_{1}}+e^{\left(2 \sigma_{S}^{s}+\epsilon\right) T_{2}}\right),
$$

(iv) Furthermore, $\tilde{\lambda}$ introduced in (iii) is such that $\forall \lambda_{1} \in[m \tilde{\lambda}, M]$ and $\forall \lambda_{2} \in$ $[-M,-m \tilde{\lambda}], \tau_{J}\left(\lambda_{1}\right) \tau_{J}\left(\lambda_{2}\right)<0$.

The (iv) from the Theorem has a meaning that the function $\tau_{J}$ changes its sign inside a "small" interval containing zero and does not change its sign outside the "small" interval in a "bigger" interval.

\subsection{Two lemmas and the proof of the Theorem}

In order to prove Theorem 2.2, we shall use estimates (4) from S.Schecter [11] and the roughness properties of exponential dichotomies.

Let $X_{\lambda}(t, s)$ be the principal matrix solution of the equation

$$
\dot{u}=\mathcal{B}_{\lambda}\left(q_{\lambda}(t)\right) u
$$


From assumption $H_{2}$ it follows that $X_{\lambda}(t, s)$ has an exponential dichotomy on $(-\infty, 0]$ with projection operators $P_{\lambda}^{u}(t)$ and $P_{\lambda}^{s}(t), P_{\lambda}^{u}(t)=I d-P_{\lambda}^{s}(t)$. It implies that there exists a constant $K>0$, such that $\forall \lambda \in \Lambda, X_{\lambda}(t, s)$ satisfies the following inequalities:

$$
\begin{aligned}
\left|X_{\lambda}(s, t) P_{\lambda}^{u}(t)\right| \leq K e^{-\left(\sigma_{R}^{u}+\epsilon / 2\right)(t-s)}, & s \leq t \leq 0 \\
\left|X_{\lambda}(t, s) P_{\lambda}^{s}(s)\right| \leq K e^{\left(\sigma_{R}^{s}-\epsilon / 2\right)(t-s)}, & s \leq t \leq 0
\end{aligned}
$$

Likewise, $X_{\lambda}(t, s)$ has an exponential dichotomy on $[0 ; \infty)$ with the same constant $K$. Hence, for sufficiently large $T_{1}$ and some positive $C_{T_{1}}, u_{\lambda}$ satisfies

$$
u\left(-T_{1}\right)=C_{T_{1}} P_{\lambda}^{u}\left(-T_{1}\right) \chi_{R}^{u}
$$

where $C_{T_{1}}$ is positive and bounded away from zero if $T_{1}$ is sufficiently large. This is a consequence of the fact that $P_{\lambda}^{u}(t) \rightarrow P_{\lambda}^{u}, P_{\lambda}^{u}$ is the spectral projection of $\mathcal{B}_{\lambda}$ corresponding to the eigenvalue $\sigma_{R}^{u}(\lambda)$, and $\mathcal{R} P_{\lambda}^{u}(t)=\operatorname{span}\left\{u_{\lambda}(t)\right\}$ for $t \in(-\infty, 0]$ (see p. $106[5]$ ). Thus, if $T_{1}$ is sufficiently large, then

$$
\left|P_{\lambda}^{u}(t) \chi_{R}^{u}\right| \geq \frac{1}{2}, t \in\left(-\infty,-T_{1}\right]
$$

A similar statement holds for $w_{\lambda}$.

From (4) it follows that for some constants $C_{1}, C_{2}$ which do not depend on $\lambda$ and $J$, the following estimate holds:

$$
\begin{aligned}
\left\|\mathcal{B}_{\lambda}\left(q_{\lambda}(t)\right)-\mathcal{B}_{\lambda}^{J}\left(\tilde{q}_{\lambda}^{J}(t)\right)\right\| & \leq C_{1}\left(\left|q_{\lambda}^{J}(t)-\tilde{q}_{\lambda}^{J}(t)\right|+\left|\tilde{\alpha}_{\lambda}^{J}\right|\right) \\
& \leq C_{2}\left(e^{-2 \sigma_{S}^{u} T_{1}}+e^{-2 \sigma_{S}^{s} T_{2}}\right) .
\end{aligned}
$$

Then we intend to use roughness properties of the exponential dichotomy to compare solutions of the linear equation

$$
\dot{x}=\mathcal{B}_{\lambda}^{J}\left(\tilde{q}_{\lambda}^{J}(t)\right) x
$$

with those of equation (9).

We introduce $B(t)$ by:

$$
B(t)=\left\{\begin{aligned}
D_{1} F\left(\tilde{q}_{\lambda}^{J}(t), \tilde{\alpha}_{\lambda}^{J}, \lambda\right), & t \in\left[-T_{1}, 0\right], \\
D_{1} F\left(\tilde{q}_{\lambda}^{J}\left(-T_{1}\right), \tilde{\alpha}_{\lambda}^{J}, \lambda\right), & t \in\left(-\infty,-T_{1}\right] .
\end{aligned}\right.
$$


Obviously $B(t)=\mathcal{B}_{\lambda}^{J}\left(\tilde{q}_{\lambda}^{J}(t)\right)$ for $t \in\left[-T_{1}, 0\right]$, and for some constant $C_{3}$ independent of $\lambda$ and $J$ we have

$$
\left\|B(t)-\mathcal{B}_{\lambda}\left(q_{\lambda}(t)\right)\right\| \leq C_{3}\left(e^{-2 \sigma_{S}^{u} T_{1}}+e^{-2 \sigma_{S}^{s} T_{2}}\right), t \in \mathbb{R}_{-} .
$$

Let $\tilde{X}_{\lambda}(t, s)$ be for $t, s \in \mathbb{R}_{-}$the principal matrix solution of the equation

$$
\dot{y}=B(t) y
$$

If $T_{1}, T_{2}$ are sufficiently large then

$$
\begin{aligned}
e^{-2 \sigma_{S}^{u} T_{1}}+e^{2 \sigma_{S}^{s} T_{2}} & \leq \frac{\sigma_{R}^{u}}{4 C_{3} K^{2}} \\
K C_{3}\left(e^{-2 \sigma_{S}^{u} T_{1}}+e^{-2 \sigma_{S}^{s} T_{2}}\right) & \leq \frac{\epsilon}{4}
\end{aligned}
$$

Then it follows from the roughness theorem [6] that $\tilde{X}_{\lambda}(t, s)$ satisfies an exponential dichotomy on $(-\infty, 0]$ with projections $\tilde{P}_{\lambda}^{u}(t), \tilde{P}_{\lambda}^{s}(t)$ satisfying, for some $t \in(-\infty, 0], \mathcal{N}\left(\tilde{P}_{\lambda}^{u}(t)\right)=\mathcal{R}\left(\tilde{P}_{\lambda}^{s}(t)\right)=\mathcal{N}\left(P_{\lambda}^{u}(t)\right)$ and

$$
\begin{aligned}
\left|\tilde{X}_{\lambda}(s, t) \tilde{P}_{\lambda}^{u}(t)\right| \leq K_{1} e^{-\sigma_{R}^{u}(t-s)}, & s \leq t \leq 0 \\
\left|\tilde{X}_{\lambda}(t, s) \tilde{P}_{\lambda}^{s}(s)\right| \leq K_{1} e^{\sigma_{R}^{s}(t-s)}, & s \leq t \leq 0 .
\end{aligned}
$$

with constant $K_{1} \geq \frac{5 K^{2}}{2}$. Moreover, there is a positive constant $C_{4}$, which can be taken independently of $J$ and $\lambda$ such that

$$
\left\|\tilde{P}_{\lambda}^{u}(t)-P_{\lambda}^{u}(t)\right\| \leq C_{4}\left(e^{-2 \sigma_{S}^{u} T_{1}}+e^{2 \sigma_{S}^{s} T_{2}}\right), t \in \mathbb{R}_{-} .
$$

Furthermore, it is a consequence of (12) that for $T_{1}$ sufficiently large

$$
\left|\tilde{P}_{\lambda}^{u}(t) \chi_{R}^{u}\right| \geq \frac{1}{4}, t \in\left(-\infty,-T_{1}\right]
$$

Applying similar arguments we conclude that $\tilde{X}_{\lambda}(t, s)$ has an exponential dichotomy also on $[0 ; \infty)$ with the same constants $K_{1}, \sigma_{R}^{u}$ and $\sigma_{R}^{s}$.

Now we state a lemma in which solutions of (5a-5b) are compared with solutions of $(7 a-7 b)$. 
Lemma 2.3 Let $H_{1}-H_{5}$ hold, $J=\left[-T_{1}, T_{2}\right], T_{1}, T_{2}$ sufficiently large, and $\tilde{y}_{\lambda}^{J}$, $\tilde{z}_{\lambda}^{J}$ be solutions of (7a-7b) and $u_{\lambda}, w_{\lambda}$ be solutions of (5a-5b). Then for some constant $C$, which does not depend on $\lambda$ and $J$,

$$
\begin{aligned}
& \left|u_{\lambda}(0)-\tilde{y}_{\lambda}^{J}(0)\right| \leq C\left(e^{\left(-\sigma_{R}^{u}+\sigma_{R}^{s}\right) T_{1}}+e^{-2 \sigma_{S}^{u} T_{1}}+e^{-2 \sigma_{S}^{s} T_{2}}\right), \\
& \left|w_{\lambda}(0)-\tilde{z}_{\lambda}^{J}(0)\right| \leq C\left(e^{\left(-\sigma_{R}^{u}+\sigma_{R}^{s}\right) T_{2}}+e^{-2 \sigma_{S}^{u} T_{1}}+e^{-2 \sigma_{S}^{s} T_{2}}\right) .
\end{aligned}
$$

Proof . We consider $t \leq 0$ and estimate both terms in the right hand side of the inequality

$$
\begin{aligned}
\left|u_{\lambda}(0)-\tilde{y}_{\lambda}^{J}(0)\right| \leq & \left|\tilde{y}_{\lambda}^{J}(0)-\frac{\tilde{X}_{\lambda}\left(0,-T_{1}\right) \tilde{P}^{u}\left(-T_{1}\right) \chi_{R}^{u}}{\left|\tilde{X}_{\lambda}\left(0,-T_{1}\right) \tilde{P}^{u}\left(-T_{1}\right) \chi_{R}^{u}\right|}\right| \\
& +\left|u_{\lambda}(0)-\frac{\tilde{X}_{\lambda}\left(0,-T_{1}\right) \tilde{P}^{u}\left(-T_{1}\right) \chi_{R}^{u}}{\left|\tilde{X}_{\lambda}\left(0,-T_{1}\right) \tilde{P}^{u}\left(-T_{1}\right) \chi_{R}^{u}\right|}\right|
\end{aligned}
$$

We consider the first term. By definition we have

$$
\begin{aligned}
\tilde{y}_{\lambda}^{J}(0)= & \frac{\tilde{X}_{\lambda}\left(0,-T_{1}\right) \tilde{P}_{\lambda}^{u}\left(-T_{1}\right) \chi_{R}^{u}}{\left|\tilde{X}_{\lambda}\left(0,-T_{1}\right) \tilde{P}_{\lambda}^{u}\left(-T_{1}\right) \chi_{R}^{u}+\tilde{X}_{\lambda}\left(0,-T_{1}\right) \tilde{P}_{\lambda}^{s}\left(-T_{1}\right) \chi_{R}^{u}\right|} \\
& +\frac{\tilde{X}_{\lambda}\left(0,-T_{1}\right) \tilde{P}_{\lambda}^{s}\left(-T_{1}\right) \chi_{R}^{u}}{\left|\tilde{X}_{\lambda}\left(0,-T_{1}\right) \tilde{P}_{\lambda}^{u}\left(-T_{1}\right) \chi_{R}^{u}+\tilde{X}_{\lambda}\left(0,-T_{1}\right) \tilde{P}_{\lambda}^{s}\left(-T_{1}\right) \chi_{R}^{u}\right|} .
\end{aligned}
$$

From (14) and (16), we see that

$$
\begin{gathered}
\left|\tilde{X}_{\lambda}\left(0,-T_{1}\right) \tilde{P}_{\lambda}^{u}\left(-T_{1}\right) \chi_{R}^{u}\right| \geq \frac{1}{4 K_{1}} e^{\sigma_{R}^{u} T_{1}}, \\
\left|\tilde{X}_{\lambda}\left(0,-T_{1}\right) \tilde{P}_{\lambda}^{s}\left(-T_{1}\right) \chi_{R}^{u}\right| \leq K_{1} e^{\sigma_{R}^{s} T_{1}},
\end{gathered}
$$

and if $T_{1}$ is sufficiently large then

$$
\left|\tilde{X}_{\lambda}\left(0,-T_{1}\right) \tilde{P}_{\lambda}^{s}\left(-T_{1}\right) \chi_{R}^{u}\right| \leq \frac{1}{2}\left|\tilde{X}_{\lambda}\left(0,-T_{1}\right) \tilde{P}_{\lambda}^{u}\left(-T_{1}\right) \chi_{R}^{u}\right| .
$$

Then there is a constant $C_{1}$ such that

$$
\left|\frac{\tilde{X}_{\lambda}\left(0,-T_{1}\right) \tilde{P}_{\lambda}^{s}\left(-T_{1}\right) \chi_{R}^{u}}{\left|\tilde{X}_{\lambda}\left(0,-T_{1}\right) \tilde{P}_{\lambda}^{u}\left(-T_{1}\right) \chi_{R}^{u}+\tilde{X}_{\lambda}\left(0,-T_{1}\right) \tilde{P}_{\lambda}^{s}\left(-T_{1}\right) \chi_{R}^{u}\right|}\right| \leq C_{1} e^{\left(\sigma_{R}^{s}-\sigma_{R}^{u}\right) T_{1}} .
$$


Similarly,

$$
\begin{aligned}
& \left|\frac{\tilde{X}_{\lambda}\left(0,-T_{1}\right) \tilde{P}_{\lambda}^{u}\left(-T_{1}\right) \chi_{R}^{u}}{\left|\tilde{X}_{\lambda}\left(0,-T_{1}\right) \tilde{P}_{\lambda}^{u}\left(-T_{1}\right) \chi_{R}^{u}+\tilde{X}_{\lambda}\left(0,-T_{1}\right) \tilde{P}_{\lambda}^{s}\left(-T_{1}\right) \chi_{R}^{u}\right|}-\frac{\tilde{X}_{\lambda}\left(0,-T_{1}\right) \tilde{P}_{\lambda}^{u}\left(-T_{1}\right) \chi_{R}^{u}}{\left|\tilde{X}_{\lambda}\left(0,-T_{1}\right) \tilde{P}_{\lambda}^{u}\left(-T_{1}\right) \chi_{R}^{u}\right|}\right| \\
& \quad=\left|\frac{\left|\tilde{X}_{\lambda}\left(0,-T_{1}\right) \tilde{P}_{\lambda}^{u}\left(-T_{1}\right) \chi_{R}^{u}\right|}{\left|\tilde{X}_{\lambda}\left(0,-T_{1}\right) \tilde{P}_{\lambda}^{u}\left(-T_{1}\right) \chi_{R}^{u}+\tilde{X}_{\lambda}\left(0,-T_{1}\right) \tilde{P}_{\lambda}^{s}\left(-T_{1}\right) \chi_{R}^{u}\right|}-1\right| \\
& \quad \leq C_{1} e^{\left(\sigma_{R}^{s}-\sigma_{R}^{u}\right) T_{1}} .
\end{aligned}
$$

Thus, we have shown that there is a constant $C_{2}$ such that

$$
\left|\tilde{y}_{\lambda}^{J}(0)-\frac{\tilde{X}_{\lambda}\left(0,-T_{1}\right) \tilde{P}^{u}\left(-T_{1}\right) \chi_{R}^{u}}{\left|\tilde{X}_{\lambda}\left(0,-T_{1}\right) \tilde{P}^{u}\left(-T_{1}\right) \chi_{R}^{u}\right|}\right| \leq C_{2} e^{\left(\sigma_{R}^{s}-\sigma_{R}^{u}\right) T_{1}} .
$$

Now we estimate the second term in the right hand side of (17). Using (15) and (11) we obtain that

$$
\left|\frac{\tilde{P}_{\lambda}^{u}\left(-T_{1}\right) \chi_{R}^{u}}{\left|\tilde{P}_{\lambda}^{u}\left(-T_{1}\right) \chi_{R}^{u}\right|}-\frac{u_{\lambda}\left(-T_{1}\right)}{\left|u_{\lambda}\left(-T_{1}\right)\right|}\right| \leq C_{4}\left(e^{-2 \sigma_{S}^{u} T_{1}}+e^{2 \sigma_{S}^{s} T_{2}}\right)
$$

and, hence, for $T_{1}, T_{2}$ sufficiently large

$$
0<\left\langle\frac{\tilde{P}_{\lambda}^{u}\left(-T_{1}\right) \chi_{R}^{u}}{\left|\tilde{P}_{\lambda}^{u}\left(-T_{1}\right) \chi_{R}^{u}\right|}, \frac{u_{\lambda}\left(-T_{1}\right)}{\left|u_{\lambda}\left(-T_{1}\right)\right|}\right\rangle .
$$

Since $\operatorname{span}\left\{\tilde{X}_{\lambda}\left(t,-T_{1}\right) \tilde{P}_{\lambda}^{u}\left(-T_{1}\right) \chi_{R}^{u}\right\}=\mathcal{R} \tilde{P}_{\lambda}^{u}(t)$ and $\operatorname{span}\left\{u_{\lambda}(t)\right\}=\mathcal{R} P_{\lambda}^{u}(t)$, implies that

$$
\left\langle\tilde{X}_{\lambda}\left(t,-T_{1}\right) \tilde{P}^{u}\left(-T_{1}\right) \chi_{R}^{u}, u_{\lambda}(t)\right\rangle \neq 0
$$

for all $t \in\left[-T_{1}, 0\right]$. Thus, (19) implies by continuity of solutions that

$$
\left\langle\tilde{X}_{\lambda}\left(0,-T_{1}\right) \tilde{P}_{\lambda}^{u}\left(-T_{1}\right) \chi_{R}^{u}, u_{\lambda}(0)\right\rangle>0
$$

and since $\mathcal{R} \tilde{P}_{\lambda}^{u}(0)$ and $\mathcal{R} P^{u}(0)$ are one-dimensional, we obtain using (15) that

$$
\left|\frac{\tilde{X}_{\lambda}\left(0,-T_{1}\right) \tilde{P}^{u}\left(-T_{1}\right) \chi_{R}^{u}}{\left|\tilde{X}_{\lambda}\left(0,-T_{1}\right) \tilde{P}^{u}\left(-T_{1}\right) \chi_{R}^{u}\right|}-u_{\lambda}(0)\right| \leq C_{4}\left(e^{-2 \sigma_{S}^{u} T_{1}}+e^{2 \sigma_{S}^{s} T_{2}}\right) .
$$


Combining (18) and (20), we obtain for some constant $C$ that

$$
\left|u_{\lambda}(0)-\tilde{y}_{\lambda}^{J}(0)\right| \leq C\left(e^{\left(-\sigma_{R}^{u}+\sigma_{R}^{s}\right) T_{1}}+e^{-2 \sigma_{S}^{u} T_{1}}+e^{2 \sigma_{S}^{s} T_{2}}\right) .
$$

Similarly we obtain

$$
\left|w_{\lambda}(0)-\tilde{z}_{\lambda}^{J}(0)\right| \leq C\left(e^{\left(-\sigma_{R}^{u}+\sigma_{R}^{s}\right) T_{2}}+e^{-2 \sigma_{S}^{u} T_{1}}+e^{-2 \sigma_{S}^{s} T_{2}}\right),
$$

which completes the proof.

We shall use the following lemma to solve $\tau_{J}(\lambda)=0$.

Lemma 2.4 Let $\varphi: \mathbb{R} \rightarrow \mathbb{R}$ be $C^{2}$ and $\varphi_{J}: \mathbb{R} \rightarrow \mathbb{R}$ be $C^{0}, 0<b<1, L \geq 0$ and $\Lambda=\left[-\frac{b}{L}, \frac{b}{L}\right]$. Assume that

(i) $\varphi(0)=0$,

(ii) $\left|\varphi^{\prime \prime}(\lambda)\right| \leq L$ for $\lambda \in \Lambda$,

(iii) $\left|\varphi^{\prime}(0)\right| \geq b>0$,

(iv) $\left|\varphi_{J}(\lambda)-\varphi(\lambda)\right| \leq \delta<\frac{b^{2}}{4 L}, 0<\delta, \lambda \in \Lambda$.

Then there is $\tilde{\lambda},|\tilde{\lambda}| \leq \frac{4 \delta}{b}$, such that $\varphi_{J}(\tilde{\lambda})=0$, and $\varphi_{J}(\lambda) \neq 0$ for $\lambda \in$ $\left[-\frac{b}{L},-\frac{4 \delta}{b}\right] \cup\left[\frac{4 \delta}{2}, \frac{b}{L}\right]$.

Proof . From (i)-(iii), it follows that for $\lambda \in \Lambda,|\varphi(\lambda)| \geq|\lambda|(b-|\lambda| L / 2)$. By (iii), $\varphi(\lambda)$ has different signs for $\lambda \in\left[-\frac{b}{L}, 0\right)$ and for $\lambda \in\left(0, \frac{b}{L}\right]$. It follows from (iv) that $\left|\varphi_{J}(\lambda)\right| \geq|\lambda|(b-|\lambda| L / 2)-\delta$, and that if $\lambda \in\left[\frac{4 \delta}{b}, \frac{b}{L}\right]$ or $\lambda \in\left[-\frac{b}{L},-\frac{4 \delta}{b}\right]$ then $\left|\varphi_{J}(\lambda)\right| \geq \delta>0$. Moreover, $\varphi_{J}(\lambda)$ has different sign in these two intervals.

Proof of Theorem 2.2. The first two statements of Theorem 2.2 follow from Theorem 2.1.

To prove (iii) we apply Lemma 2.4 with $\varphi=\tau$ and $\varphi_{J}=\tau_{J}$. By $H_{5}, \tau(0)=0$. The mapping $\lambda \mapsto D_{1} F\left(q_{\lambda}(t), 0, \lambda\right)$ is $C^{2}$. Consequently, $u_{\lambda}$ and $w_{\lambda}$ are $C^{2}$ in $\lambda$, hence we can choose the constant $L$ in Lemma 2.4 as $L=\sup _{\lambda \in \Lambda}\left\{\tau^{\prime \prime}(\lambda)\right\}$. 
Hypothesis $H_{6}$ gives that assumption (iii) in Lemma 2.4 is satisfied, if we choose $b=\tau^{\prime}(0)$. From Lemma 2.3, we obtain there is a constant $C$ such that

$$
\left|\tau_{J}(\lambda)-\tau(\lambda)\right| \leq C\left(e^{\left(-\sigma_{R}^{u}+\sigma_{R}^{s}\right) T_{1}}+e^{-2 \sigma_{S}^{u} T_{1}}+e^{2 \sigma_{S}^{s} T_{2}}\right) .
$$

If we choose $\delta=C\left(e^{\left(-\sigma_{R}^{u}+\sigma_{R}^{s}\right) T_{1}}+e^{-2 \sigma_{S}^{u} T_{1}}+e^{2 \sigma_{S}^{s} T_{2}}\right)$ then we satisfy condition (iv) from Lemma 2.4 if we take $T_{1}$ and $T_{2}$ sufficiently large.

Part (iii) of Theorem 2.2 follows then from the estimate for $\tilde{\lambda}$ (Lemma 2.4) and the estimate (4) for $\tilde{\alpha}_{\tilde{\lambda}}^{J}$. Finally, (iv) follows from Lemma 2.4 with $m=\frac{4 \tau_{J}(0)}{\tau^{\prime}(0)}$ and $M=\frac{\tau^{\prime}(0)}{L}$.

Remark: one may ask how the accuracy in the computation of the eigenvectors $\chi_{R}^{u}$ and $\chi_{R}^{s}$ influences the error in computations of the test function $\tau_{J}$. Fortunately, instead of the vectors $\chi_{R}^{u}$ and $\chi_{R}^{s}$ in the formulation of the boundary value problems (7a) and (7b), we can take any unit vectors $\chi_{1}$ and $\chi_{2}$, satisfying the conditions $\left|P_{\lambda}^{u}(-t) \chi_{1}\right|>0,\left|P_{\lambda}^{s}(t) \chi_{2}\right|>0, t \in[T, \infty), \forall \lambda \in \Lambda$ and for $T$ sufficiently large. These conditions are similar to (12).

\section{Numerical results}

Finally, we report on the numerical simulations obtained for system (3). We have used the continuation package AUTO86 [8]. First, we solve for an orbit satisfying the boundary conditions $x\left(-T_{1}\right)=\delta \chi^{u}$, where $\delta>0$ is a small parameter using the homotopy as described in [4]. We begin with continuation in $T_{1}$ starting from $T_{1}=0.1, \delta=0.01$ using approximate parameter values for a homoclinic orbit, namely $\beta=0.125, I=0.6$. At $T_{1}=12.54027$, the output shows that $x(0-) \in$ $\Sigma=\left\{\left(s_{1}, s_{2}\right) \in \mathbb{R}^{2}, s_{1}=3.446276\right\}$. Then, we solve for an orbit satisfying the boundary conditions $x\left(T_{2}\right)=\delta \chi^{s}$ and $x(0+) \in \Sigma$ again using the homotopy. At $T_{2}=8.32174$, the output shows that $x(0+) \in \Sigma$. We observe that there is a gap of -0.15 between $x(0-)$ and $x(0+)$ in $\Sigma$. An adjustment in $I$ eliminates the gap and produces a numerical solution of $P_{2}$.

At $\beta=0.125, I=0.6635044, T_{1}=12.86013, T_{2}=8.481780$ we have a good approximation of a homoclinic orbit. Then we perform continuation in $\delta$, which measures the distance from the points $x\left(-T_{1}\right)$ and $x\left(T_{2}\right)$ to the equilibrium $p(\lambda)$. We obtain at $\delta=10^{-5}$ that $T_{1}=32.307, T_{2}=18.72196, \beta=0.125, I=$ 
0.6635038. This numerical solution will be used to perform a two-parameter continuation in $(\beta, I)$.

We note that if the function $v(t)$ satisfies $\dot{v}(t)=\mathcal{B}_{\lambda}^{J}\left(\tilde{q}_{\lambda}^{J}(t)\right) v(t)$ then $v_{1}(t)=$ $v(t) e^{-\sigma t}$ is a solution of the modified variational equation

$$
\dot{v}_{1}(t)=\mathcal{B}_{\lambda}^{J}\left(\tilde{q}_{\lambda}^{J}(t)\right) v_{1}(t)-\sigma v_{1}(t)
$$

We exploit this to compensate for the exponential decay of the norms of the solutions of (7a), (7b) as $t \rightarrow \pm \infty$ and avoid loss of precision due to round off errors in the numerical experiments adjusting the parameter $\sigma$. We add the modified linear variational equation to the set of equations and solve them numerically. Then we perform a two-parameter continuation in $(\beta, I)$ while monitoring the test function $\tau_{J}$. Output shows the zero of the test function (8), which gives the location of the twist bifurcation:

$$
\left(\beta^{*}, I^{*}\right)=(0.4198656,0.6389036) .
$$

It turns out that at these critical parameter values the symmetric eigenvalues are $\alpha_{S}=0.350$ and $\beta_{S}=-0.642$. The eigenvalues in the asymmetric direction are $\alpha_{R}=0.612$ and $\beta_{R}=-2.999$. This is in agreement with the numbers as given in $[1]$.

In Figure 1, $\left|\beta-\beta^{*}\right|+\left|I-I^{*}\right|$ is plotted in the logarithmic scale versus $T_{1}$, fixing $T_{2}$ at 30.215 . The slope is equal to -1.345 differing from the slope -0.7 , which is the expected rate of convergence of the numerical approximation to the homoclinic orbit, by -0.645 .

In Figure 3, $\left|\beta-\beta^{*}\right|+\left|I-I^{*}\right|$ is plotted in the logarithmic scale versus $T_{2}$, fixing $T_{1}$ at 30.38638 . The slope is equal to -1.63 differing from the slope -1.284 , which is the expected rate of convergence of the numerical approximation to the homoclinic orbit, by -0.346 . Thus these examples suggest that the true convergent rate might be

$$
\left|I^{*}-\tilde{I}\right|+\left|\beta^{*}-\tilde{\beta}\right|=O\left(e^{\left(-\alpha_{R}+\beta_{R}\right) T_{1}}+e^{\left(-\alpha_{R}+\beta_{R}\right) T_{2}}+e^{\left(-2 \alpha_{S}+\beta_{S}\right) T_{1}}+e^{\left(2 \beta_{S}-\alpha_{S}\right) T_{2}}\right) .
$$

and estimate (15) can be improved. We conjecture that this can be done using a better pointwise estimate in (13). 


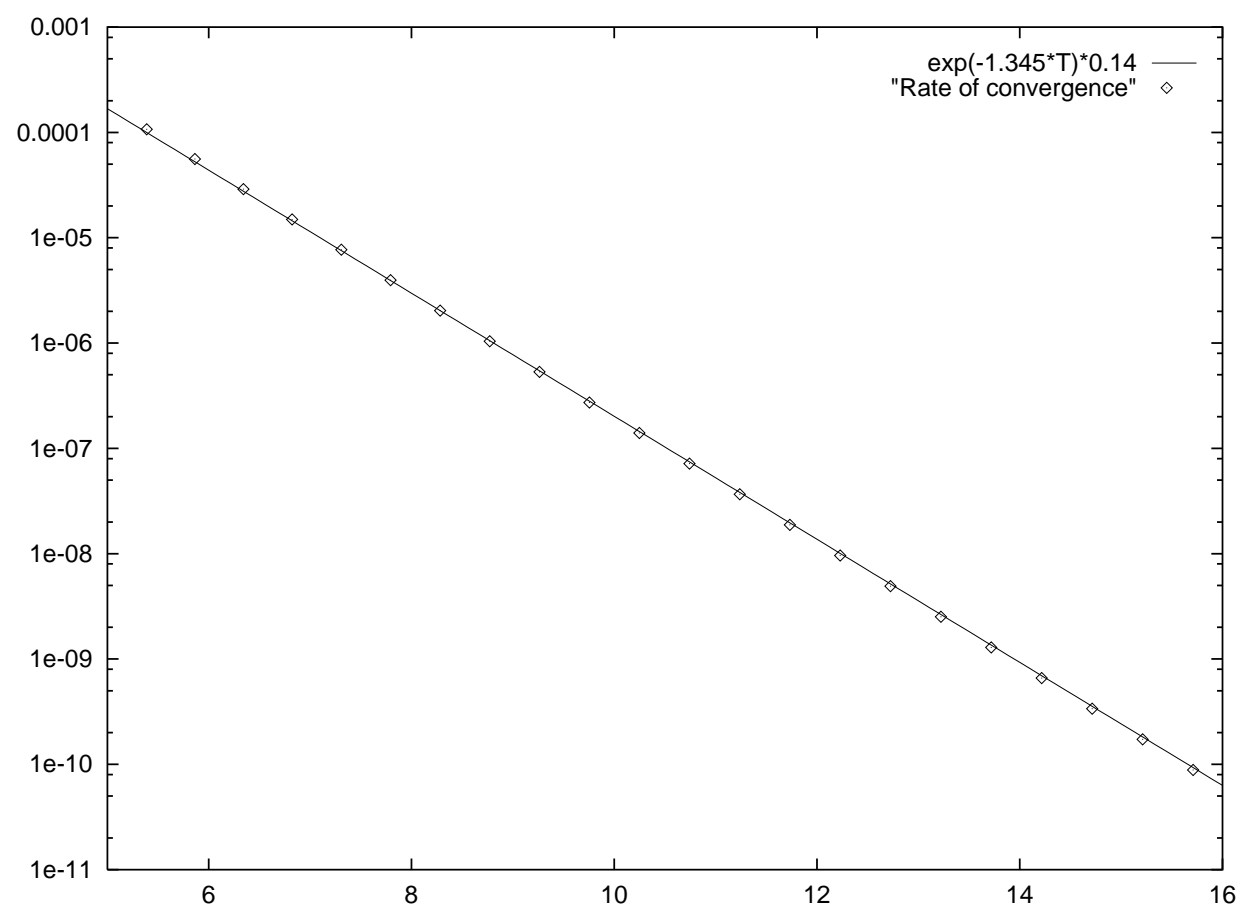

Figure 1: Graph of $\left|\beta^{*}-\beta\right|+\left|I^{*}-I\right|$ versus $T_{1}$ with the logarithmic scale in the vertical axis, $T_{2}=30.215$. 


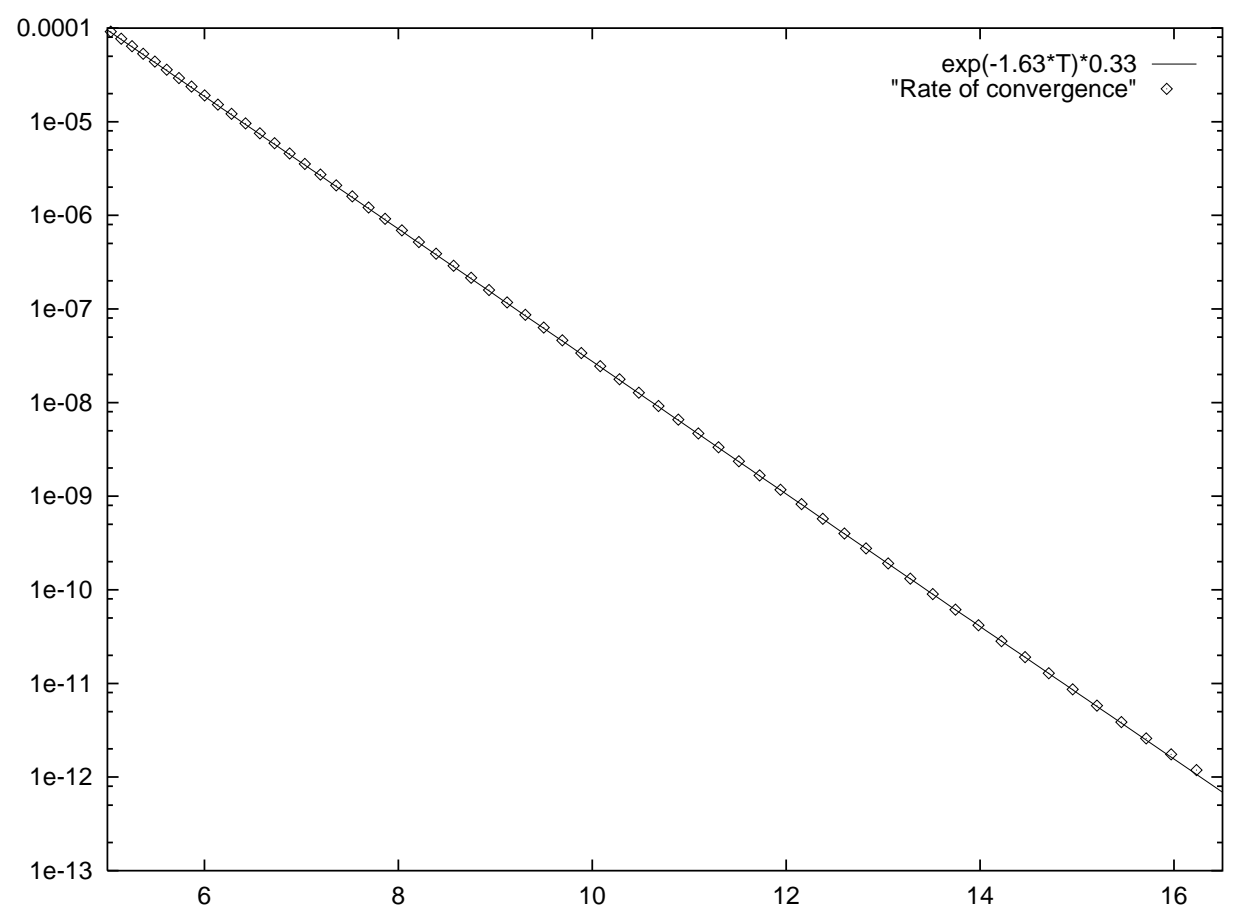

Figure 2: Graph of $\left|\beta^{*}-\beta\right|+\left|I^{*}-I\right|$ versus $T_{2}$ with the logarithmic scale in the vertical axis, $T_{1}=30.38638$. 
In the next experiment, we examine the following system depending on three parameters $(\beta, I, \gamma)$ :

$$
\begin{aligned}
& \dot{s}_{1}=s_{2}, \\
& \dot{s}_{2}=\frac{1}{3+\beta}\left(I-s_{2}\left(1+\gamma \cos \left(s_{1}\right)\right)-\sin \left(s_{1}\right) \cos \left(r_{1}\right)\right) \\
& \dot{r}_{1}=r_{2} \\
& \dot{r}_{2}=\frac{1}{\beta}\left(\gamma s_{2} \sin \left(s_{1}\right) \sin \left(r_{1}\right)-r_{2}\left(1+\gamma \cos \left(s_{1}\right) \cos \left(r_{1}\right)\right)-\sin \left(r_{1}\right) \cos \left(s_{1}\right)\right) .
\end{aligned}
$$

This equations results from a resistively and capacitively shunted junction model, see [7], here written in the symmetric coordinates. The terms multiplying $\gamma$ model the flow of unpaired electrons through the junction. At $\gamma=0$ equations (21) coincide with equations (3). We performed the continuation of the homoclinic twist bifurcations in the space of three parameters $(\beta, I, \gamma)$ for $\gamma \in[-0.261,13.48]$ (see Figure 3).

\section{Conclusions}

This paper presents a method for detecting homoclinic twist points for $Z_{2}$-symmetric vector fields. The resulting AUTO driver allows to continue twisted homoclinic orbits satisfying certain conditions in three parameters. Using this driver, a homoclinic twist point has been detected in the system of two coupled Josephson junctions.

As it has been noted in [1], the occurrence of twist points is independent of the number of the junctions in the array. The representation of the group $S_{n}$, the symmetry group of the equations governing the evolution of an array of $n$ globally coupled junctions in $\mathbb{R}^{2 n}$, admits decomposition in the direct sum of irreducible representations. The $(n-1)$-dimensional irreducible representation and onedimensional identity representation have multiplicity two in this decomposition. Thus in the symmetric coordinates the matrix of the variational equation along the symmetric homoclinic solution $q_{\lambda}(t)$ is a block diagonal matrix

$$
\left(\begin{array}{cccc}
\mathcal{A}_{\lambda}\left(q_{\lambda}(t)\right) & & & 0 \\
& \mathcal{B}_{\lambda}\left(q_{\lambda}(t)\right) & & \\
0 & & \ddots & \\
& & & \mathcal{B}_{\lambda}\left(q_{\lambda}(t)\right)
\end{array}\right)
$$




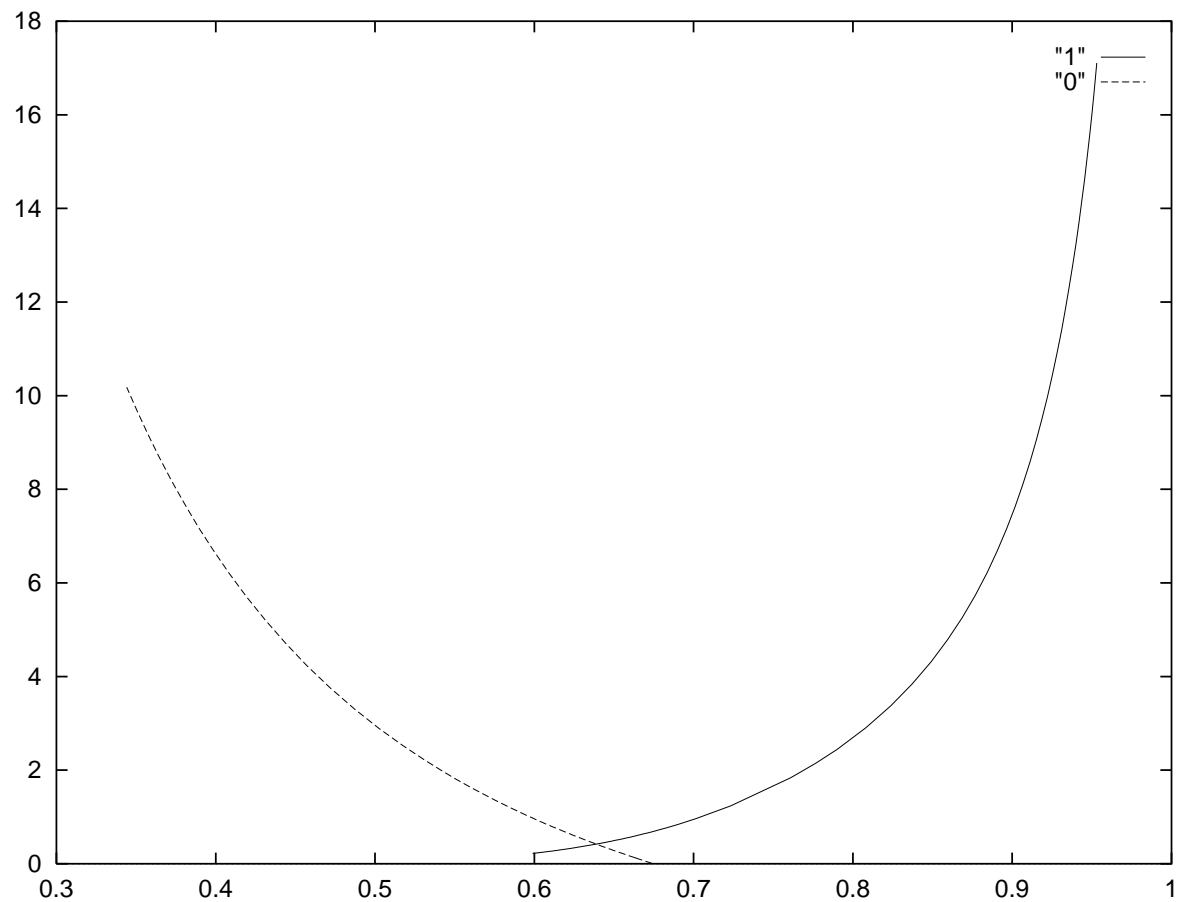

Figure 3: Bifurcation diagram for equation $(21)$ in $(I, \beta)$-plane. Along curve " 0 " there is a branch of symmetric homoclinic connections, at $\gamma=0$. Homoclinic twist bifurcations occur along curve " 1 ", the parameter $\gamma$ is changing in the interval $[-0.261,13.48]$. 
where $\mathcal{A}_{\lambda}\left(q_{\lambda}(t)\right), \mathcal{B}_{\lambda}\left(q_{\lambda}(t)\right)$ are $2 \times 2$ blocks. Hence, we can use the same test function $\tau_{J}$ for detecting homoclinic twist points in the system of $n$-coupled Josephson junctions.

The general case for a smooth vector field $F: \mathbb{R}^{n} \times \mathbb{R}^{2} \rightarrow \mathbb{R}^{n}$ is $C^{3}$ equivariant with respect to the action of some finite group $\Gamma \in O(n)$, and admitting a branch of symmetric homoclinic orbits, requires additional treatment. The codimension-two homoclinic twist bifurcation occurs only if the subspace $\dot{q}_{0}(0)^{\perp} \cap \operatorname{dim} T_{q_{0}(0)} W^{s} \cap T_{q_{0}(0)} W^{u}$ is an irreducible subspace and it has multiplicity two in the decomposition of the state space into irreducible subspaces. Then, $D_{1} F\left(q_{\lambda}, 0, \lambda\right)$ has a block-diagonal structure in the corresponding coordinates, and we can supply a test function using solutions of the corresponding linear variational equation.

\section{References}

[1] D.G.Aronson, S.A. van Gils, M.Krupa. Homoclinic twist bifurcation with $Z_{2}$ symmetry. J. Nonlinear Science 4 (1994) 195-219.

[2] D.G.Aronson, M.Golubitsky, M.Krupa. Coupled arrays of Josephson junctions and bifurcation of maps with $S_{N}$ symmetry. Nonlinearity 4 (1991) 861902.

[3] W.-J. Beyn. The numerical computation of connected orbits in dynamical systems. IMA Journal of Numerical Analysis 9 (1990) 379-405.

[4] A.R.Champneys, Yu.A.Kuznetsov. and B.Sandstede A numerical toolbox for homoclinic bifurcation. International journal of bifurcation and chaos in applied sciences and engineering, 6(6), 1996, p. 867-888.

[5] E.A. Coddington, N. Levinson. Theory of Ordinary Differential Equations. McGraw-Hill. New York, 1955.

[6] W.A.Coppel. Dichotomies in Stability Theory. Lect. Notes in Math., 629. Springer, Berlin, 1978.

[7] R.H.Cushman, J.Sanders. Limit cycles in the Josephson equation, SIAM J. Math. Anal., 17 (1986), 495-511. 
[8] E.J.Doedel. AUTO: A program for automatic bifurcation analysis of autonomous systems. Cong. Num. 30 (1981) 265-284.

[9] K.J.Palmer. Exponential dichotomies and transversal homoclinic points. J. Differential equations 55 (1984) 255.

[10] B.Sandstede. Numerical computation of homoclinic flip-bifurcations. 1995. In preparation.

[11] S. Schecter. Rate of convergence of numerical approximation to homoclinic bifurcation points. IMA Journal of Numerical Analysis 15 (1995) 23-60. 